\title{
Chemometric evaluation of biogenic amines in commercial fruit juices
}

\author{
Raffaella Preti $^{1} \cdot$ Roberta Bernacchia $^{1} \cdot$ Giuliana Vinci $^{1}$
}

Received: 25 November 2015 / Revised: 11 April 2016 / Accepted: 2 May 2016

(C) Springer-Verlag Berlin Heidelberg 2016

\begin{abstract}
The profile and level of 11 biogenic amines were evaluated in commercial fruit juices (apple, grapefruit, orange and pineapple) and fruit nectars (apricot, peach and pear) by HPLC/FD after dansyl chloride derivatization. The biogenic amine most present in nectars is cadaverine, followed by putrescine, spermidine and spermine. Fruit juices showed a wider variability in biogenic amines profile and level, with the highest total content in orange juices. The application of chemometric tools as hierarchical cluster analysis and principal component analysis on the biogenic amine profiles of the juice samples succeeded in grouping juices on the basis of the fruit of origin. A mathematical model with high predictive ability for fruit juices classification was obtained by linear discriminant analysis: orange $(100 \%)$, pineapple $(100 \%)$, grapefruit $(80 \%)$ and apple (70\%). This study represents the first description of biogenic amines content in these beverages. These compounds are well-known important quality parameters and demonstrated to have also a characteristic profile depending on the fruit of origin.
\end{abstract}

Keywords Biogenic amines - Fruit juices - Fruit nectars · Chemometric analysis $\cdot$ Characterization

Raffaella Preti

raffaella.preti@uniroma1.it

1 Laboratory of Commodity Science, Department of Management, Sapienza University of Rome, Via del Castro Laurenziano 9, 00164 Rome, Italy

\section{Introduction}

Fruit juices are recognized as valuable contributors to a healthy diet, being good sources of vitamins, minerals and other beneficial micronutrients, such as carotenoids, limonoids and lycopene. Fruit content and good production technology are the main quality parameters of such fruit preparations.

As defined in EU Council Directive 2001/112/EC, fruit juices consist of $100 \%$ pure juice made from the flesh of fresh fruit or from concentrates and contain no flavorings, colors, preservatives or any other added ingredients; fruit concentrated juice is obtained by reconstituting concentrated fruit juice with potable water; fruit nectars are obtained by adding water with or without the addition of sugars, to the fruit juices, fruit purée and/or concentrated fruit purée reaching a fruit content in the range $25-99 \%$; and minimum juice content varies according to the fruit in question.

Fruit-based processed products, in particular juices, are being increasingly consumed in modern societies. Fruit juice and nectars consumption in the EU stood at almost 10 billion liters in 2014. Hundred percentages of juice accounted for approaching two-thirds of the EU fruit juice and nectars market total in 2014, equivalent to 6.2 billion liters [1].

The adulteration of fruit juice is quite common due to various factors such as high product demand, high price, short harvest season and shortage of production in some regions. Mixing with other juices is also done to compensate the negative effects of low-quality raw materials and/or processing. Types of juice adulterations include the dilution with water, the addition of sugar solution, citric and tartaric acids, colorants and of cheaper and widely available juices originating from other fruits, mainly from grapefruit, apples, peach or pear [2-4]. 
Numerous methods for authenticity control in fruitbased products have been developed [5, 6]. These studies work on the significant variance of several specific fruit chemical components. Among them, phenolic compounds are mainly used for chemotaxonomic purposes. In fact, depending on species, cultivar, ripening stage, environmental conditions and postharvest treatments the polyphenolic content of fruit allows to detect fraudulent admixtures [7]. Together with phenolic compounds, also anthocyanins, organic acids, amino acids and sugars are frequently used for the estimation of fruit content as well as the control of authenticity, thanks to chemotaxonomic characteristics $[8$, 9].

Biogenic amines are formed by microbial decarboxylation of the corresponding amino acids, and several amines are naturally present in foods. Some of them are reported to be specific of some plant species, where they exert essential metabolic functions, and therefore can be used in an explorative study as a potential chemotaxonomic index [10, $11,12]$.

The presence of biogenic amines in different foods can be related to the hygienic-sanitary conditions and spoilage as to be considered a marker of food quality $[12,13]$. Furthermore, biogenic amines can induce several negative physiological reactions and so the knowledge of their levels in foods is of great importance for consumer's health and for the formulation of diets. In particular, patients with cancer should be exposed to diets with reduced levels of polyamines [14], and individuals under MAOI drugs (antituberculosis and antidepressant drugs) should avoid foods rich in monoamines [15].

Information on the types and levels of bioactive amines in fruits and products is not yet explored in depth. Up to now, in international literature, only two works describe the presence of biogenic amines in fruit juices, in particular in orange juices [10, 16]. Vieira et al. [10] analyzed the profile and level of biogenic amines in orange juices and orange soft drinks; among the nine amines found putrescine was the predominant, followed by synephrine and spermidine. Similar results have been obtained by Basheer et al. [16] in the analysis of orange juices of different brands. No literature papers investigated the profile of biogenic amines in other juices except two articles on polyamines [17, 18].

Several methods for the determination of BAs in foods have been developed, and the most used involve high-performance liquid chromatography (HPLC) coupled with UV or FD detector. Analytical methods usually consist in an acid extraction followed by a derivatization step. Also methods in UHPLC and capillary zone electrophoresis (CZE) are reported [19].

The determination of the profile and the levels of eleven biogenic amines in fruit juices and nectars carried out in the present work, has two main goals: the quality and safety assessment of these beverages and the evaluation of the suitability of these compounds as fruit-type differentiation marker. For this purpose, principal component analysis (PCA), cluster analysis (CA) and linear discriminant analysis (LDA) were chosen as the most frequently employed methods for initial exploration of data and sample clustering [20].

\section{Materials and methods}

\section{Chemicals}

Perchloric acid, acetone (analytical grade), acetonitrile (HPLC grade), glucose, phenolphthalein and Fehling solution, as well as the other reagents, were purchased from Sigma-Aldrich (Milan, Italy). Ultrapure water $\left(18.2 \mathrm{M} \Omega \mathrm{cm}\right.$, resistivity at $\left.25{ }^{\circ} \mathrm{C}\right)$ was obtained by a Milli-Q (Millipore, Bedford, MA, USA). The eleven biogenic amines studied were: ethylamine (ETA), methylamine (MEA), histamine (HIS), serotonin (SER), spermine (SPM), spermidine (SPD), agmatine (AGM), putrescine (PUT), $\beta$-phenylethylamine ( $\beta$-PEA), cadaverine (CAD) and tyramine (TYM), all of which were supplied by Supelco (Bellefonte, PA, USA) as well as the derivatizating agent (dansyl chloride) and the internal standard (1,7-diaminoheptane) (IS).

\section{Instrumentation}

HPLC separations were developed in a system consisting in a LC-10 ATVP binary HPLC pump with a RF-10AXL fluorescence detector (Shimadzu, Kyoto, Japan). The injector was fitted with a 20- $\mu 1$ loop. The chromatographic data were collected and processed using Class-VP software (Shimadzu). The analytical column was a $100 \times 4.6 \mathrm{~mm}$ I.D. Kinetex C18 column (particle size $2.6 \mu \mathrm{m}$ ), with KrudKatcher C18, an integrated $0.5-\mu \mathrm{m} 316$ stainless steel depth filter that removes microparticulates from the flow stream with minimal contributions to system dead volume $(0.2 \mu \mathrm{L})$ (all supplied by Phenomenex, Torrance, CA, USA). Analytical column temperature was kept at $50{ }^{\circ} \mathrm{C}$ by a CT 10AS oven (Shimadzu, Kyoto, Japan). The maximum operating pressure on the system was 400 bar.

\section{Samples}

Samples of commercial fruit nectar (31) and concentrated fruit juice (43) were purchased from local markets and stored at room temperature before analysis. Samples were produced by the major Italian fruit juice brands (Table 1). Types of fruit products were chosen as to be the most representative of the market, for fruit nectar were apricot, peach 
Table 1 Commercial fruit juice concentrates and nectars considered in this study

\begin{tabular}{lllll}
\hline Fruit & $\begin{array}{l}\text { Declared } \\
\text { minimum fruit } \\
\text { concentration (\%) }\end{array}$ & Brands & Family & Genus \\
\hline Fruit nectar & & & & \\
Apricot & 50 & 7 & & Prunus \\
Peach & 50 & 6 & & Pyrus \\
Peach & 70 & 6 & Rosaceae & \\
Pear & 50 & 6 & & Malus \\
Pear & 70 & 6 & & Citrus \\
Fruit juices & & 10 & Rosaceae & \\
Apple & 100 & 10 & Rutaceae & Ananas \\
Grapefruit & 100 & 11 & & Bromeliaceae \\
Orange & 100 & 12 & & \\
Pineapple & 100 & & & \\
\hline
\end{tabular}

and pear, while for concentrated fruit juice were apple, pineapple grapefruit and orange. As described in EU Council Directive 2001/112/EC the fruit nectars were labeled with the minimum content of fruit in percentage required for each fruit nectar, only peach and pear nectars are present in the market with two different percentages 50 and $70 \%$, and in this study, they were evaluated separately.

\section{Analytical methods}

The samples were analyzed for biogenic amines, $\mathrm{pH}$, titratable acidity, total sugars and soluble solids (Table 2).

\section{Determination of general quality parameters}

$\mathrm{pH}$ was measured with a digital $\mathrm{pH}$ meter, HI110 model (Hanna Instruments, USA), after calibration with $\mathrm{pH} 4.0$ and 7.0 standard buffers [21]. Total acidity was determined by titration with $0.1 \mathrm{~N} \mathrm{NaOH}$, standardized before use, adding phenolphthalein indicator [21]. Total sugars (reducing and nonreducing) were determined by Lane and Eynon [22] method. The clarified samples, containing equal volumes of Fehling A and B, were titrated with $0.5 \%$ glucose solution. Inversion for nonreducing sugars was necessary to calculate the total sugars [21]. The levels of soluble solids, expressed as degrees Brix, were determined using a refractometer, model RL1-PZO (Warszawa, Poland), equipped with a thermometer. The results were corrected for the temperature when the determinations were made at temperatures other than $20^{\circ} \mathrm{C}$ [21].

\section{Determination of biogenic amines}

Biogenic amines in fruit nectars were determined according to a previously optimized method [23]. Briefly, $2.5 \mathrm{~mL}$ of fruit nectar previously added with IS $(0.5 \mathrm{~mL}, 0.5 \mathrm{~g} / \mathrm{L})$ was extracted with $7.5 \mathrm{~mL}$ of $\mathrm{HClO}_{4} 0.4 \mathrm{M}$ and centrifuged at $2000 \mathrm{rpm}$ for $5 \mathrm{~min}$. Supernatant was filtered through a $0.20-\mu \mathrm{m}$ membrane Millipore filter, and sediment was added with $1.5 \mathrm{~mL}$ of $\mathrm{HClO}_{4} 0.4 \mathrm{M}$ and centrifuged again for $3 \mathrm{~min}$. The second extract was then filtered and added to the first. The final volume was adjusted to $10 \mathrm{~mL}$ with $\mathrm{HClO}_{4} 0.4 \mathrm{M}$. An aliquot of $1 \mathrm{~mL}$ of the final extract was then derivatized by adding $200 \mu \mathrm{L}$ of $\mathrm{NaOH} 2 \mathrm{M}, 300 \mu \mathrm{L}$ of saturated $\mathrm{NaHCO}_{3}$ solution and $2 \mathrm{~mL}$ of dansyl chloride solution $(10 \mathrm{mg} / \mathrm{mL}$ in acetone). After shaking, samples were left in the dark at $45{ }^{\circ} \mathrm{C}$ for $60 \mathrm{~min}$. The final volume was adjusted to $5 \mathrm{~mL}$ by adding acetonitrile. The dansylated extract was filtered using $0.22 \mu \mathrm{m}$ (Polypro Acrodisc, Pall Gelman Laboratory, USA) and injected into the chromatograph.

A method for the determination of biogenic amines in concentrated fruit juices was developed starting from the procedure described for wine in the previous work [23].

Table 2 Mean values (SD) of $\mathrm{pH}$, titratable acidity and total sugars of the samples analyzed

\begin{tabular}{|c|c|c|c|c|c|}
\hline Fruit & Declared minimum fruit concentration $(\%)$ & $\mathrm{pH}$ & Total acidity ${ }^{\mathrm{a}}$ & Total sugars $(\mathrm{g} / 100 \mathrm{~g})$ & Brix \\
\hline \multicolumn{6}{|l|}{ Fruit nectars } \\
\hline Apricot & 50 & $3.44(0.23)$ & $0.69(0.13)$ & $13.72(0.22)$ & $11.20(0.10)$ \\
\hline Peach & 50 & $3.86(0.27)$ & $0.37(0.10)$ & $12.03(0.10)$ & $12.30(0.21)$ \\
\hline Peach & 70 & $3.73(0.22)$ & $0.51(0.14)$ & $12.32(0.28)$ & $12.70(0.15)$ \\
\hline Pear & 50 & $3.91(0.20)$ & $0.29(0.12)$ & $(0.11)$ & $12.20(0.10)$ \\
\hline Pear & 70 & $3.68(0.19)$ & $0.39(0.11)$ & $12.40(0.13)$ & $12.80(0.22)$ \\
\hline \multicolumn{6}{|l|}{ Fruit juices } \\
\hline Apple & 100 & $3.64(0.16)$ & $0.55(0.15)$ & $11.25(0.20)$ & $11.51(0.22)$ \\
\hline Grapefruit & 100 & $3.32(0.14)$ & $1.16(0.22)$ & $9.83(0.18)$ & $10.60(0.30)$ \\
\hline Orange & 100 & $3.79(0.20)$ & $0.86(0.18)$ & $10.15(0.15)$ & $11.40(0.21)$ \\
\hline Pineapple & 100 & $3.76(0.18)$ & $0.65(0.16)$ & $10.40(0.14)$ & $13.01(0.20)$ \\
\hline
\end{tabular}

\footnotetext{
a Percent of citric acid
} 
Table 3 Concentrations ( $\mathrm{mg} / \mathrm{L}$ ) of biogenic amines in fruit nectars

\begin{tabular}{lllllll}
\hline Fruit juices & \multicolumn{6}{l}{ Mean levels* (SD) } \\
\cline { 2 - 7 } & SPD & SPM & PUT & ETA & CAD & BA tot \\
\hline Apricot 50 \% $(n=7)$ & $2.31^{\mathrm{a}}(0.21)$ & $1.89^{\mathrm{a}}(0.51)$ & $2.76^{\mathrm{a}}(2.22)$ & nd & $10.97^{\mathrm{a}}(6.45)$ & $17.90^{\mathrm{a}}(7.99)$ \\
Range & $1.97-2.52$ & $1.22-2.51$ & $1.39-7.10$ & - & $3.96-17.93$ & $10.45-28.74$ \\
Peach 50 \% $(n=7)$ & $1.58^{\mathrm{b}}(0.25)$ & $1.74^{\mathrm{a}}(0.58)$ & $2.35^{\mathrm{a}}(0.75)$ & nd & $5.80^{\mathrm{b}}(2.92)$ & $11.50^{\mathrm{b}}(3.62)$ \\
Range & $1.34-2.01$ & $1.22-2.72$ & $1.41-3.19$ & - & $1.95-10.06$ & $7.74-17.47$ \\
Peach 70 \% $(n=6)$ & $3.06^{\mathrm{c}}(0.89)$ & $1.58^{\mathrm{a}}(0.20)$ & $3.13^{\mathrm{a}}(0.52)$ & nd & $4.95^{\mathrm{b}}(0.70)$ & $12.70^{\mathrm{ab}}(1.36)$ \\
Range & $2.02-4.44$ & $1.37-1.89$ & $2.25-3.59$ & - & $4.11-6.05$ & $11.04-14.57$ \\
Pear 50 \% $(n=6)$ & $1.52^{\mathrm{b}}(0.27)$ & $1.87^{\mathrm{a}}(0.95)$ & $1.68^{\mathrm{a}}(0.60)$ & nd & $5.06^{\mathrm{b}}(3.16)$ & $10.10^{\mathrm{b}}(3.88)$ \\
Range & $1.16-1.77$ & $1.17-3.53$ & $1.11-2.68$ & - & $1.91-8.31$ & $4.23-13.21$ \\
Pear 70 \% $(n=6)$ & $2.17^{\mathrm{a}}(0.34)$ & $1.32^{\mathrm{a}}(0.11)$ & $2.83^{\mathrm{a}}(1.38)$ & $1.17(0.07)$ & $4.68^{\mathrm{b}}(1.29)$ & $12.21^{\mathrm{ab}}(2.30)$ \\
Range & $1.90-2.65$ & $1.22-1.41$ & $1.42-4.44$ & $1.12-1.23$ & $3.75-6.15$ & $7.61-13.22$ \\
\hline
\end{tabular}

$S P D$ spermidine, $S P M$ spermine, $P U T$ putrescine, ETA ethylamine, $C A D$ cadaverine, $B A$ tot total biogenic amines, $n d$ not detected

* Mean values with different letters in the same column are significantly different (Tukey's test, $p<0.05$ )
Twenty-five milliliters of fruit juice, previously added with IS $(0.5 \mathrm{~mL}, 1 \mathrm{~g} / \mathrm{L})$, was acidified by $\mathrm{HClO}_{4} 10.3 \mathrm{M}$ to reach a final acid concentration of $0.2 \mathrm{M}$ and then dansylated and quantified as described above.

The chromatographic conditions were as follows: A linear gradient was from $65 \%$ acetonitrile to $75 \%$ acetonitrile in $3.5 \mathrm{~min}$ and then increased to $100 \%$ acetonitrile in 9 min and then kept for 2 min for a total run time of $11 \mathrm{~min}$ at a constant flow rate of $0.6 \mathrm{~mL} / \mathrm{min}$. Fluorescence detection was set at $320 \mathrm{~nm}$ for excitation and $523 \mathrm{~nm}$ for emission. Identification of the biogenic amines was based on their retention times. Accuracy (recovery $>93 \%$ ) and precision (RSD $<8 \%$ ) were evaluated in triplicate using fruit juices and nectars spiked at two concentration levels of biogenic amines. Quantitation was performed using the internal standard calibration method using linear regression analysis $(r \geq 0.999)$. The limit of detection (LOD) values were between 0.002 (PUT) and $0.023 \mathrm{mg} / \mathrm{L}$ (AGM). The corresponding limit of quantification (LOQ) values were 0.006 and $0.077 \mathrm{mg} / \mathrm{L}$. The absence of any matrix effect and interferences has been also assessed.

\section{Statistical analysis}

The quantifications of biogenic amines in commercial fruit nectars and juices were carried out in triplicate. The range, mean and standard deviation (SD) for every type of sample were calculated.

Analysis of variance (ANOVA) and mean comparison by Tukey's honest significant difference (HSD) for unequal number of samples at $5 \%$ level were performed using STATGRAPHICS Centurion XV 15.2.06 (Statpoint Technologies, Inc., Warrenton, VA, USA). Chemometric data analyses (PCA, CA and LDA) were performed with VPARVUS [24].

\section{Results and discussion}

The samples were quite heterogeneous, as they were chosen from all categories of fruit beverages in the market to explore the possibility of finding differences in the samples for biogenic amines profile and content in relation to botanical origin and their processing. Therefore, in the data analysis and presentation the samples are divided into two groups, the concentrated fruit juices and the fruit nectars.

The $\mathrm{pH}$, total acidity values, total sugars and soluble solids were measured for all the samples, and results are reported in Table 2. The accordance of these parameters with data reported in the literature $[10,25]$ confirms the good quality of the commercial products considered.

\section{Profile and levels of biogenic amines in fruit nectars}

Among the 11 biogenic amines analyzed, only five were detected in the 31 samples analyzed (Table 3). Spermidine, spermine, putrescine and cadaverine were found in all fruit nectar samples, while ethylamine was determined only in three pear samples with a fruit content above $70 \%$. Methylamine, $\beta$-phenylethylamine, serotonin, tyramine, agmatine and histamine were not detected in any samples.

Spermidine and spermine occur ubiquitously in plant kingdom, together with their precursor putrescine, so their presence was expected also in fruit derivatives.

Cadaverine is formed from the decarboxylation of lysine and has been previously reported in other vegetable products such as tomato products [26]. This diamine resulted to be the predominant amine in all fruit nectars. In apricot nectar in particular, cadaverine had significantly higher level $(p<0.05)$, contributing to the total level of amines with $61 \%$ followed by putrescine with $15 \%$. Cadaverine and putrescine show a large variability in different apricot 
Table 4 Levels $(\mathrm{mg} / \mathrm{L})$ of biogenic amines in fruit juice concentrates

\begin{tabular}{|c|c|c|c|c|c|c|c|c|c|c|}
\hline \multirow[t]{2}{*}{ Fruit juices } & \multicolumn{10}{|c|}{ Mean levels* (SD) } \\
\hline & SPD & SPM & PUT & ETA & CAD & TYM & MEA & SER & HIS & BA tot \\
\hline $\begin{array}{l}\text { Apple } \\
\quad(n=10)\end{array}$ & $0.46^{\mathrm{a}}(0.15)$ & $0.43^{\mathrm{a}}(0.22)$ & $1.02^{\mathrm{a}}(0.35)$ & $\begin{array}{l}0.15^{\mathrm{a}} \\
\quad(0.15)\end{array}$ & $\begin{array}{l}2.30^{\mathrm{a}} \\
\quad(1.53)\end{array}$ & $\begin{array}{l}0.67^{\mathrm{a}} \\
\quad(0.50)\end{array}$ & nd & nd & nd & $5.35^{\mathrm{a}}(1.01)$ \\
\hline Range & $0.24-0.67$ & $0.24-0.99$ & $0.59-1.68$ & nd- -0.41 & $0.55-4.27$ & nd-1.6 & nd & nd & nd & $3.67-6.54$ \\
\hline $\begin{array}{l}\text { Pineapple } \\
\quad(n=12)\end{array}$ & $\begin{array}{l}4.25^{\mathrm{b}} \\
\quad(0.91)\end{array}$ & $\begin{array}{l}2.50^{\mathrm{b}} \\
\quad(0.38)\end{array}$ & $\begin{array}{l}1.79^{\mathrm{a}, \mathrm{b}} \\
(0.16)\end{array}$ & $\begin{array}{l}0.51^{\mathrm{a}} \\
\quad(0.42)\end{array}$ & $\begin{array}{l}1.21^{\mathrm{b}} \\
\quad(1.22)\end{array}$ & $\begin{array}{l}0.87^{\mathrm{a}} \\
\quad(0.86)\end{array}$ & $\begin{array}{l}1.03^{\mathrm{a}} \\
\quad(0.48)\end{array}$ & $\begin{array}{l}4.68^{\mathrm{a}} \\
\quad(1.88)\end{array}$ & $\begin{array}{l}2.44^{\mathrm{a}} \\
\quad(1.59)\end{array}$ & $\begin{array}{l}20.13^{\mathrm{b}} \\
(2.02)\end{array}$ \\
\hline Range & $2.55-5.41$ & $1.53-3.17$ & $1.53-1.98$ & $0.22-1.65$ & nd-3.14 & nd-1.93 & nd-1.88 & $1.54-7.17$ & nd-4.61 & $22.92-16.81$ \\
\hline $\begin{array}{l}\text { Grapefruit } \\
\quad(n=10)\end{array}$ & $1.41^{\mathrm{c}}(0.45)$ & $0.38^{\mathrm{a}}(0.06)$ & $\begin{array}{c}10.08^{\mathrm{b}} \\
(4.11)\end{array}$ & $\begin{array}{l}9.85^{\mathrm{b}} \\
(2.58)\end{array}$ & $\begin{array}{l}1.00^{\mathrm{b}} \\
(0.64)\end{array}$ & nd & $\begin{array}{l}0.28^{\mathrm{b}} \\
(0.46)\end{array}$ & $\begin{array}{l}0.50^{\mathrm{b}} \\
(1.03)\end{array}$ & $\begin{array}{l}0.31^{\mathrm{b}} \\
(0.58)\end{array}$ & $\begin{array}{r}23.87^{\mathrm{b}} \\
(5.02)\end{array}$ \\
\hline Range & $1.03-2.11$ & $0.32-0.50$ & 7.17-20.80 & $6.21-12.98$ & $0.38-2.28$ & - & nd-1.17 & nd-2.82 & nd-1.74 & $17.88-35.79$ \\
\hline $\begin{array}{l}\text { Orange } \\
\qquad(n=11)\end{array}$ & $\begin{array}{l}2.76^{\mathrm{d}} \\
\quad(0.50)\end{array}$ & $0.68^{\mathrm{a}}(0.32)$ & $\begin{array}{l}45.51^{\mathrm{c}} \\
\quad(8.35)\end{array}$ & $\begin{array}{r}32.23^{\mathrm{c}} \\
(4.73)\end{array}$ & nd & nd & $\begin{array}{l}1.87^{\mathrm{c}} \\
\quad(0.85)\end{array}$ & $\begin{array}{l}0.18^{\mathrm{b}} \\
\quad(0.44)\end{array}$ & $\begin{array}{l}0.46^{\mathrm{b}} \\
\quad(0.41)\end{array}$ & $\begin{array}{l}83.98^{\mathrm{c}} \\
\quad(10.29)\end{array}$ \\
\hline Range & $2.04-3.66$ & $0.37-1.37$ & $\begin{array}{r}34.70- \\
60.97\end{array}$ & $\begin{array}{r}24.06- \\
38.64\end{array}$ & - & - & nd-2.72 & nd-1.35 & nd-1.32 & $67.90-98.23$ \\
\hline
\end{tabular}

SPD spermidine, SPM spermine, PUT putrescine, ETA ethylamine, CAD cadaverine, TYM tyramine, MEA methylamine, SER serotonin, HIS histamine, $n d$ not detected

* Means are calculated using nd (not detected) equal to 0 . Mean values with different letters in the same column are significantly different (Tukey's test, $p<0.05$ )

nectar brands, with concentrations ranging from 3.96 to $17.93 \mathrm{mg} / \mathrm{L}$ and 1.39 to $7.10 \mathrm{mg} / \mathrm{L}$, respectively.

The statistical analysis of the data did not indicate significative differences $(p>0.05)$ among fruits for spermine and putrescine, while spermidine was significantly lower in peach $50 \%$ and pear $50 \%$ and higher in peach $70 \%$. The mean total amine levels varied from $10.1 \mathrm{mg} / \mathrm{L}$ in pear $50 \%$ to $17.9 \mathrm{mg} / \mathrm{L}$ in apricot, with no significant difference $(p>0.05)$ among peach and pear samples, and among apricot, peach $70 \%$ and pear $70 \%$ samples.

In the absence of previous studies on the biogenic amines content in the fresh fruits, some hypothesis can be postulated on the origin of the predominance of cadaverine in apricot nectars and its wide variability, as well as for putrescine. These data can be related to the high presence of the precursor amino acids in this type of fruit, to the high variability of raw materials quality and of the technological processes used in the production. In fact, several cultivars and different degrees of ripening or different geographical origins of the fruits with different climatic conditions and cultivation practices can be used for the production of apricot nectars [9]. Furthermore, in nonfermented foods, the presence of biogenic amines above a certain level can be considered as indicative of undesired microbial activity. Therefore, the amine level could be used as an indicator of microbial spoilage [27].

Interestingly, comparing the biogenic amines content in fruit nectars made with the same fruit (peach or pear) but with different fruit content (50 or $70 \%$ ), no significant differences were highlighted in all the amines detected except for spermidine, which its level in the nectars belonging to the $70 \%$ category was significantly higher than in the $50 \%$. This relation between fruit content and spermidine level can be attributed to the origin of this amine mainly from its presence in the fresh fruit and not from decarboxylation activities following the processing.

\section{Profile and levels of biogenic amines in concentrated fruit juices}

The profile and the levels of biogenic amines in the 43 samples of concentrated fruit juices analyzed were quite different from those in fruit nectars, both for the fruit and for the technology used in their production (Table 4). Fruit nectars derive from fruits belonging to the same botanical family (Rosaceae), while the concentrated fruit juices were taxonomically different apart from grapefruit and orange that were of the same genus.

In this kind of fruit products, 9 of the 11 amines studied were detected. As for fruit nectars, spermine, spermidine and putrescine were determined in every sample analyzed, as typical amines of vegetable products, while agmatine and $\beta$-phenylethylamine were never detected.

Putrescine was the most abundant amine in fruit juices, with the highest $(p<0.05)$ mean concentration of $45.5 \mathrm{mg} / \mathrm{L}$ in orange juices and lowest in apple juices $(1.02 \mathrm{mg} / \mathrm{L})$.

Ethylamine occurred in every sample except for 4 apple juice brands, with orange and apple again with the highest and the lowest level, respectively. On the contrary, 
cadaverine was never found in orange juices, while the mean level in apple juices $(2.30 \mathrm{mg} / \mathrm{L})$ was significantly the highest.

Tyramine occurred in few samples, in the $80 \%$ of apple and in $45 \%$ of pineapple juices but never in citrus fruit. Methylamine was determined in every sample of orange and pineapple juices, the highest level was in orange $(p<0.05)$, while this volatile amine occurred only in the $50 \%$ of grapefruit and never in apple juice samples. Serotonin was present at the higher mean level $(p<0.05)$, and in every pineapple juice object of the study, it was also detected in the $20 \%$ of both citrus juices but in none of the apple juice samples.

Histamine, one of the most dangerous amine for human health [10], had the highest level $(p<0.05)$ in pineapple juice samples $(2.44 \mathrm{mg} / \mathrm{L})$ where it was detected in every sample as well as for orange juice samples, but at a lower concentration $(0.46 \mathrm{mg} / \mathrm{l})$, furthermore grapefruit juices presented $>30 \%$ of samples with histamine above the LOQ $(0.19 \mathrm{mg} / \mathrm{L})$.

Results for orange juices are in accordance with previously reported data $[10,16]$, which are the only references found in recent literature for the analysis of biogenic amines in fruit juices. Cipolla et al. [17] analyzed some fruit juices and fresh fruits for only polyamines content, finding comparable results except for spermine that was not detected in any sample and cadaverine that was found only in citrus fruit juices; pineapple juices were not considered in this study. Regarding the analysis of the raw material fresh fruits, Nishibori et al. [28] analyzed only apple and orange and only for polyamines.

For the data on pineapple juices we can only refer to the work by Santiago-Silva et al. [11] on biogenic amines in tropical fruits, where is reported the presence of serotonin as the prevalent amine, while histamine, tyramine and cadaverine were not detected in the fresh pineapple fruit. This can suggest the conclusion that these three latter amines are produced in relation to low-quality fruit, during the processing or during storage, and can be therefore an index of quality of the product, being histamine and tyramine the amines most dangerous for human health [10].

The mean total levels of amines varied from 5.35 (apple juices) to $83.98 \mathrm{mg} / \mathrm{L}$ (orange juices), and this evidence makes fruit juices and fruit nectars interesting contributors to biogenic amines daily intake, considering the large quantities that can be consumed for their health-promoting effects. It has been reported that an intake $>40 \mathrm{mg}$ biogenic amines per meal can be considered potentially toxic [29]; however, not all amines are equally toxic and additional risk factors such as amine oxidase-inhibiting drugs, alcohol and gastrointestinal diseases may play an important role in determining the threshold for bioactive amines toxicity.

\section{Multivariate data analysis}

The different profile of biogenic amines in the fruit juice concentrates has suggested the hypothesis that some of the amines detected could be typical of a specific botanical family and therefore could be explored as potential markers of authenticity of the taxonomic origin.

For this purpose, unsupervised pattern recognition techniques such as cluster analysis (CA) and principal components analysis (PCA) were used to evaluate the data matrices in order to highlight a natural grouping among samples. These techniques were applied to all the samples object of this study, but appreciable results were obtained only for concentrated fruit juices. Starting from these results, an linear discriminate analysis (LDA) model was constructed to provide a mathematical model to classify juice samples according to fruit composition.

CA and PCA were carried after autoscaling on the biogenic amines data matrix of concentrated fruit juices. For CA, sample similarities were calculated on the basis of squared Euclidean distance, and the Ward hierarchical agglomerative method was used to establish clusters. Results are displayed as a dendrogram in Fig. 1 and as a bidimensional plot of sample scores and loadings in the space (defined by the first two principal components) in Fig. 2. The dendrogram shows four clusters at a similarity level of 0.6: Cluster A contains only some apple juice samples, cluster B contains the remaining apple juices and grapefruit juices, cluster $\mathrm{C}$ contains only orange juices, and cluster $\mathrm{D}$ contains only pineapple juices. Increasing the level of similarity from 0.6 to 0.7 , cluster B can be distinguished to two subclusters B' and B" that consist of apple juices and grapefruit juices samples, respectively. It is interesting to highlight the behavior of grapefruit and orange that belong to the same botanical family Citrus, but are classified into two different clusters, while grapefruit juices are closer to apple, showing a similar, unexpected, biogenic amines profile.

In PCA (Fig. 2a), the first two principal components (PC1 and PC2) accounted for $74 \%$ of the total variability. The variables (Fig. 2b) that contributed more to the PC1 $(47.2 \%)$ were spermine, serotonin, spermidine, histamine. The PC2 $(26.8 \%)$ was associated with cadaverine, methylamine, putrescine and ethylamine. In accordance with the results of cluster analysis, in PCA scores plot (Fig. 2a), pineapple and orange juices were clearly separated between them and from the other groups (grapefruit and apple juices). These last fruit juices are gathered in two different groups, but they are not definitively separated, as to be clearly classified.

The natural separation achieved by the application of these two chemometric techniques to the biogenic amines data allowed a good classification of juices 
Fig. 1 Dendrogram of cluster analysis of biogenic amines in fruit juices from concentrate. $A P$ apple, $G R$ grapefruit, $O R$ orange, $P I$ pineapple

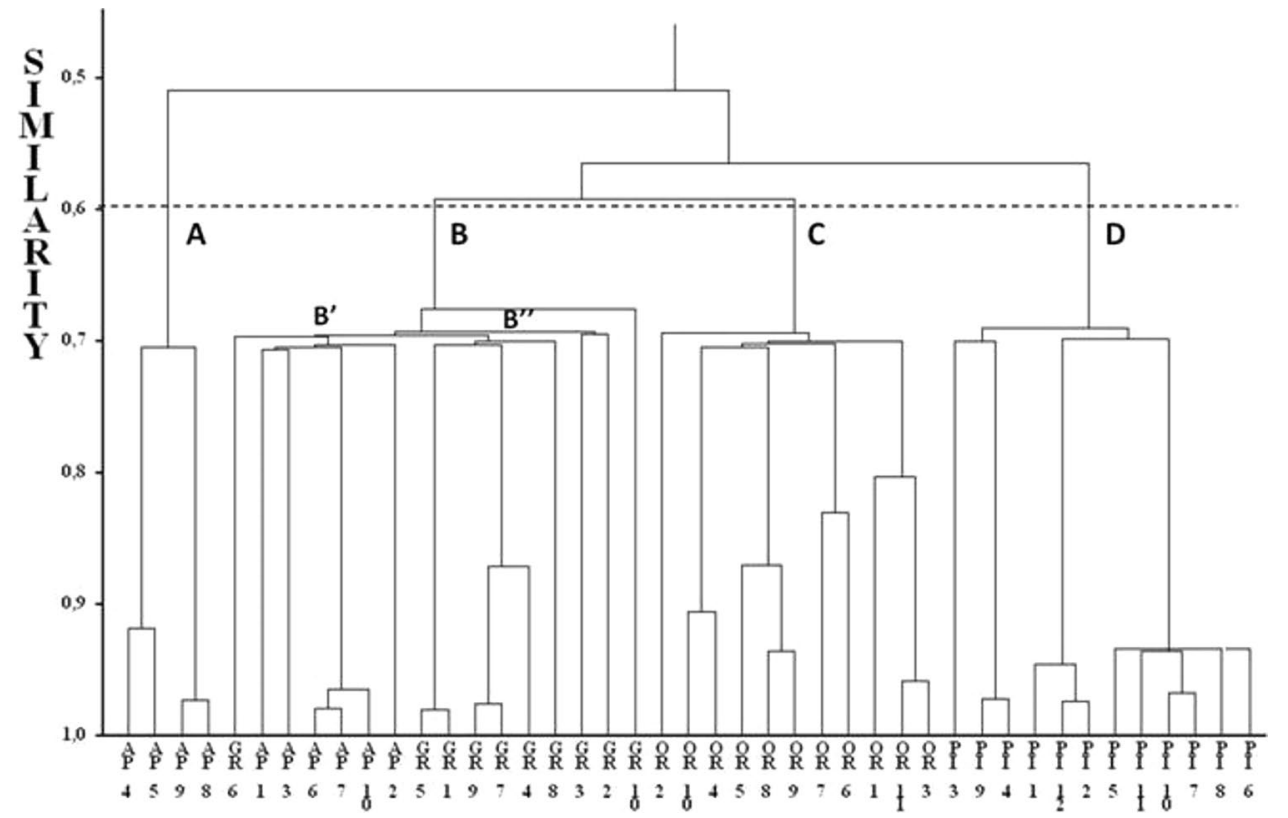

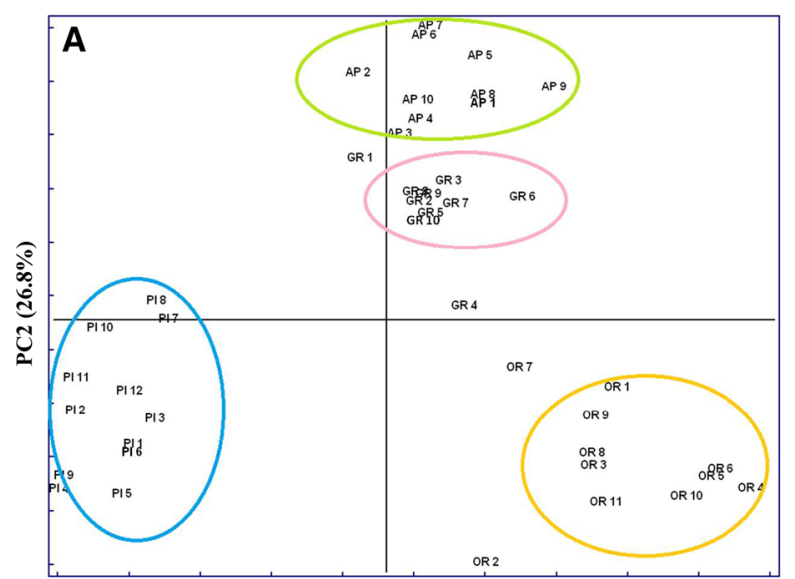

PC1 (47.2\%)

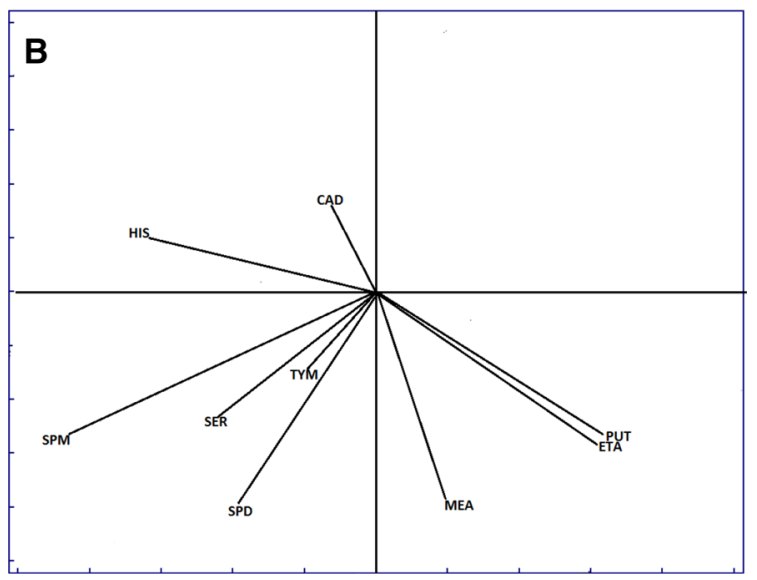

Fig. 2 Principal component scores (a) and loadings (b) plots of fruit juice concentrates. $P I$ pineapple, $O R$ orange, $G R$ grapefruit, $A P$ apple

Table 5 Results of the classification ability of the LDA model for juices according to different fruit composition

Correct $(\%)$ Apple Pineapple Grapefruit Orange

Assigned fruit category

Classification matrix

True fruit category

Apple $\quad 95.58$

411

Pineapple 100.00

Grapefruit 94.88

Orange $\quad 100.00$

Total $\quad 97.62$

Prediction matrix

True fruit category

\begin{tabular}{lrcccc} 
Apple & 70.00 & 7 & & 3 & \\
Pineapple & 100.00 & & 12 & & \\
Grapefruit & 80.00 & 2 & & 8 & 11 \\
Orange & 100.00 & & & & 11 \\
Total & 87.5 & 9 & 12 & 11 & \\
\hline
\end{tabular}

samples. To further explore the juices differentiation according to fruit composition and biogenic amines profile, an LDA model was constructed. LDA was performed on the original $\mathrm{N}$ samples using the scores of the samples on the first four PCs for the previous model as variables.

Classification results of LDA model were presented in terms of recognition and prediction abilities. Recognition ability represents a percentage of successfully classified samples in the training set. Prediction ability is a 
percentage of correctly classified samples in the test set by using the model developed during the training step.

Validation of the LDA model was carried out following leave-one-out cross-validation (LOOCV) procedure. This validation procedure is performed by removing one sample at a time, to be used as a test set, while the remaining samples are formed as a training set. The LDA classification results of all samples from four groups are shown in Table 5 .

Correct classification (recognition and prediction abilities of 97.62 and $87.50 \%$, respectively) for all samples in both training and validation step was achieved.

In the training set, some samples from apples were incorrectly assigned as grapefruit juices, and also some grapefruit juices were classified as apple juices. In the prediction set, the prediction ability for apple juices category was $70 \%$ because 3 juices from apple were incorrectly assigned as grapefruit juices. In the same way, 2 grapefruit juices were classified as apple juices, with a resulting prediction ability for grapefruit class belonging of $80 \%$. Juices from orange and pineapple were correctly classified $(100 \%)$ both in classification and in prediction matrix.

This pattern of recognition and prediction ability is in good correspondence with the results of the two explanatory analyses (CA and PCA) conducted. Juices from apple and grapefruit were shown to be more closely related with respect to others for the content of biogenic amines.

The method proposed in this article is suitable for the discrimination of fruit juices from their biogenic amines content. About prediction abilities, the method seems to be more accurate for the discrimination of juices from orange and pineapple (100 and $100 \%)$, but satisfactory performances were also obtained for the classification of fruit juices from apple and grapefruit categories (70 and $80 \%$ ).

\section{Conclusions}

This study is the first investigation on the presence of 11 biogenic amines in fruit nectars and fruit juice concentrates. The results covered the lack of information to have a complete estimation of biogenic amines dietary intake, which is of interest due to the association of these compounds to health and diseases, especially in case of sensitive consumers, deficient or impaired by diseases or by pharmacological agents, such as monoaminoxidase inhibitor (MAOI) drugs [30].

By the application of chemometric techniques, biogenic amines profile demonstrated to be also useful for the differentiation of fruit juices according to their fruit of origin.

The biogenic amine profile analysis as marker of botanical origin could be experimented also on less common fruit products, trying to find possible characterization. This aspect will be the object of future experiments, together with the investigation on the origin of biogenic amines in fruit juices, whether from endogenous pathways or from microbiological contamination.

\section{Compliance with ethical standards}

Conflict of interest The authors declare that they have no conflict of interest.

Compliance with ethics requirements This article does not contain any studies with human or animal subjects.

\section{References}

1. AIJN-European Fruit Juice Association (2015) Liquid fruit market report. http://www.aijn.org/files/default/aijn2015-report.pdf. Accessed 11 Apr 2016

2. Fugel R, Carle R, Schieber A (2005) Quality and authenticity control of fruit purèes, fruit preparations and jams-a review. Trends Food Sci Tech 16:433-441

3. Vaclavik L, Schreiber A, Lacina O, Cajka T, Hajslova J (2012) Liquid chromatography-mass spectrometry-based metabolomics for authenticity assessment of fruit juices. Metabolomics 8:793-803

4. Boggia R, Casolino MC, Hysenaj V, Oliveri P, Zunin P (2013) A screening method based on UV-Visible spectroscopy and multivariate analysis to assess addition of filler juices and water to pomegranate juices. Food Chem 140:735-741

5. Ogrinc N, Košir IJ, Spangenberg JE, Kidrič J (2003) The application of NMR and MS methods for detection of adulteration of wine, fruit juices, and olive oil. A review. Anal Bioanal Chem 376:424-430

6. Reid LM, O’Donnell CP, Kelly JD, Downey G (2004) Preliminary studies for the differentiation of apple juice samples by chemometric analysis of solid-phase microextraction-gas chromatographic data. J Agric Food Chem 52:6891-6896

7. Abad-García B, Garmón-Lobato S, Sánchez-Ilárduya MB, Berrueta LA, Gallo B, Vicente F, Alonso-Salces RM (2014) Polyphenolic contents in Citrus fruit juices: authenticity assessment. Eur Food Res Tech 238:803-818

8. Gomez-Ariza JL, Villegas-Portero MJ, Bernal-Daza V (2005) Characterization and analysis of amino acids in orange juice by HPLC-MS/MS for authenticity assessment. Anal Chim Acta 540:221-230

9. Versari A, Parpinello G, Mattioli A, Galassi S (2008) Characterization of Italian commercial apricot juices by high-performance liquid chromatography analysis and multivariate analysis. Food Chem 108:334-340

10. Vieira SM, Theodoro KH, Glória MB (2007) Profile and levels of bioactive amines in orange juice and orange soft drink. Food Chem 100:895-903

11. Santiago-Silva P, Labanca RA, Gloria MBA (2011) Functional potential of tropical fruits with respect to free bioactive amines. Food Res Int 44:1264-1268

12. Preti R, Vieri S, Vinci G (2016) Biogenic amines profile and antioxidant properties of Italian red wines from different price categories. J Food Comp Anal 46:7-14

13. Vinci G, Antonelli ML (2002) Biogenic amines: quality index of freshness in red and white meat. Food Contr 13:519-524

14. Eliassen KA, Reistad R, Risoen U, Ronning HF (2002) Dietary polyamines. Food Chem 78:273-280 
15. Gloria MBA (2005) Bioactive amines. In: Hui YH (ed) Handbook of Food Science, Technology and Engineering. CRC Press, Melbourne, $\mathrm{p} 38$

16. Basheer C, Wong W, Makahleh A, Tameem AA, Salhin A, Saad B, Lee HK (2011) Hydrazone-based ligands for micro-solid phase extraction-high performance liquid chromatographic determination of biogenic amines in orange juice. J Chrom A 1218:4332-4339

17. Cipolla BG, Havouis R, Moulinoux JP (2007) Polyamine contents in current foods: a basis for polyamine reduced diet and a study of its long term observance and tolerance in prostate carcinoma patients. Amino Acids 33:203-212

18. Ali MA, Strömberg RE, Yngve A (2011) Polyamines in foods: development of a food database. Food Nutr Res. 55:5572-5587

19. Bedia Erim F (2013) Recent analytical approaches to the analysis of biogenic amines in food samples. TrAC 52:239-247

20. Berrueta LA, Strömberg RE, Yngve K (2007) Supervised pattern recognition in food analysis. J Chrom A 1158:196-214

21. AOAC (1995) Official methods of analysis of AOAC international (16th ed.). AOAC International, Maryland, USA

22. Lane JH, Eynon L (1923) Determination of reducing sugars by means of Fehling's solution with methylene blue as internal indicator. J Chem Soc Trans 42:32-36

23. Preti R, Antonelli ML, Bernacchia R, Vinci G (2015) Fast determination of biogenic amines in beverages by a core-shell particle column. Food Chem 187:555-562
24. Forina M, Lanteri S, Armanino C, Cerrato-Oliveiros C, Casolino C, VPARVUS: An Extendable Package of Programs for Data Explorative Analysis, Classification and Regression Analysis. Genova: Department of Chimica e Tecnologie Farmaceutiche e Alimentari, University of Genova (2010). http://www.parvus. unige.it

25. Càmara M, Diez C, Torija E (1995) Chemical characterization of pineapple juices and nectars. Principal components analysis. Food Chem 54:93-100

26. Chiacchierini E, Restuccia D, Vinci G (2006) Evaluation of two different extraction methods for chromatographic determination of bioactive amines in tomato products. Talanta 69:548-555

27. Onal A (2007) A review: current analytical methods for the determination of biogenic amines in foods. Food Chem 103:1475-1486

28. Nishibori N, Fujihara S, Akatuk T (2007) Amounts of polyamines in foods in Japan and intake by Japanese. Food Chem 100:491-497

29. Ayres JC, Mundt JO, Sandine WE (1980) Microbiology of Foods. W. H. Freeman and Co, San Francisco 543

30. Shalaby AR (1996) Significance of biogenic amines to food safety and human health. Food Res Int 29:675-690 